CERN-TH/2000-025

Imperial/TP/99-0/19

hep-th/0001170

January, 2000

\title{
Brane Descent Relations in M-theory
}

\author{
Laurent Houart \\ Theory Division, CERN \\ 1211 Genève 23, Switzerland \\ and \\ Theoretical Physics Group \\ Blackett Laboratory, Imperial College \\ London $S W^{\gamma}$ 2BZ, UK \\ 1.houart@ic.ac.uk \\ and \\ Yolanda Lozano \\ Theory Division, CERN \\ 1211 Genève 23, Switzerland \\ yolanda.lozano@cern.ch
}

\begin{abstract}
We discuss how the BPS branes of M-theory could be described as bound states of non-BPS M10-branes. This conjectured M10-brane is constructed as an unstable spacetime-filling brane in the massive eleven dimensional supergravity defined with a Killing direction, such that 1) the BPS M9-brane is obtained after the tachyonic mode of an M2-brane ending on it condenses, and 2) it gives the non-BPS D9brane of the type IIA theory upon reduction. The existence of other non-BPS M-branes is also discussed, together with their possible stabilisation within the Horava-Witten construction.
\end{abstract}

PACS: 11.24.-w; 11.27.+d

Keywords: Branes; Duality; M-theory 


\section{Introduction}

Recent progress in the understanding of the role of tachyonic excitations in unstable brane systems has led to a new framework in which D-branes appear as topological defects in the worldvolume of these unstable systems. In this framework the tachyon is considered as a Higgs field which tends to develop a stable vacuum expectation value, and the solitonic configuration that appears after the condensation is a BPS D-brane (see [1] and references therein). The process can be iterated by embedding the unstable tachyonic system onto a similar system of higher dimension, and one can derive in this manner brane descent relations.

The mechanism of tachyon condensation can be given a qualitative description by looking at the couplings in the Wess-Zumino term of the effective action corresponding to the unstable system. This analysis allows to describe as well NS-NS charged branes, in particular fundamental strings, as topological solitons in higher dimensional systems [2, 3]. In this case the tachyonic condensing charged object is extended and non-perturbative, and a more desirable quantitative description is far out of reach.

In the type IIB theory starting with an unstable system of D9, anti-D9 pairs of branes it is possible to classify the stable Dp-branes in the theory by analysing the homotopy groups of the vacuum manifold of the tachyonic field [4]. Mathematically the Dp-brane charges are classified by the K-theory groups $\tilde{K}\left(S^{9-p}\right)$, which describe the equivalence classes of pairs of vector bundles that characterise the system of coincident D9, anti-D9 branes up to creation and annihilation [4]. S-duality determines that, similarly, NS-NS branes can be classified by analysing the homotopy groups associated to the tachyonic field of systems of NS9, anti-NS9 pairs of branes [5]. The K-theory description is identical to that of D-branes, but the equivalence classes of pairs of vector bundles characterise instead coincident NS9, anti-NS9 branes.

In the type IIA theory D-brane descent relations can be derived by analysing the homotopy groups of the vacuum manifold of the tachyonic field in a system of $n$ D8, anti-D8 pairs of branes and, recalling that the D8-brane can be obtained from a non-BPS D9-brane after tachyonic condensation [6], the Type IIA Dp-branes can finally be described as bound states of spacetime-filling branes, in this way preserving all the symmetries of the ten dimensional spacetime. Mathematically the D-brane charges are classified by $K^{-1}\left(S^{9-p}\right)$ K-theory groups (see [6]).

In this paper we analyse the possibility of having brane descent relations in M-theory. We show that the brane descent relations that one expects to find by oxidation from Type IIA can be predicted through the analysis of the worldvolume effective action of a system of $n$ M9, anti-M9 pairs of 
branes. We propose as well the construction of a non-BPS M10-brane from which the BPS M9-brane is obtained after the tachyonic mode of an open M2-brane ending on it condenses. Therefore we are able to write the brane descent relations from M9, anti-M9 pairs in terms of M-theory spacetimefilling branes, thereby preserving the symmetries of the eleven dimensional theory.

The study of 9-branes and 10-branes in M-theory should be carried out in the context of massive eleven dimensional supergravity, given that the BPS M9-brane couples magnetically to the mass. It is well-known that a fully eleven dimensional Lorentz invariant massive supergravity cannot be constructed [7]. Nevertheless, the massive Type IIA supergravity of Romans 88 can be derived from eleven dimensions if the condition of eleven dimensional Lorentz invariance is relaxed and one assumes an isometric eleventh direction that is gauged in the supergravity action [9]. In this paper, we will work in the framework of this massive eleven dimensional supergravity defined with a Killing isometry. This theory is therefore only invariant under ten dimensional Lorentz transformations. Accordingly, the branes living in this massive eleven dimensional background are described by worldvolume effective actions where the Killing direction is gauged . This is also the case for the non-BPS M10-brane, as we will see.

The Born-Infeld part of the M9-brane effective action has been constructed in [10, 11], and the Wess-Zumino term, which is the relevant part for the study of brane solitons, has also been derived recently in [12]. This construction provides a description of the M9-brane in terms of a gauged sigma-model2]. The effective action of an M9, anti-M9 pair can be described by a similar but more complicated expression which contains as well explicit couplings to the tachyonic mode that must be present in this unstable system. This tachyonic excitation must appear in the spectrum of an open M2-brane stretched between the brane and the antibrane, as predicted by the duality with the type IIA theory, where the open string stretched between a D8, anti-D8 pair of branes contains this type of excitation. The effective action of the M9, anti-M9 pair can be derived by oxidating the effective action describing this D8, anti-D8 pairf. Recalling that the brane antibrane system is characterised by two field strengths (one for the brane and one for the antibrane) and that the non-trivial character of the soliton can be carried by just one of the two, it is enough to look at the couplings in the worldvolume

\footnotetext{
${ }^{1}$ Also additional terms proportional to the mass are added [9].

${ }^{2}$ In such a way that the right number of degrees of freedom of a vector multiplet is recovered [10], and the brane consistently propagates in a massive background.

${ }^{3}$ Effective actions describing type II Dp, anti-Dp pairs of branes have been constructed in [13].
} 
effective action of the brane, in our case of the M9-brane, in order to find out the topological defects that the system supports, taking into account as well that for this analysis the coupling of the tachyonic mode to the worldvolume effective action does not play any role.

Keeping this in mind we analyse in section 2 the topological defects that can occur in the worldvolume of $n$ M9, anti-M9 pairs of branes by studying the different terms that couple in the WZ part of the worldvolume effective action of a system of $n$ M9-branes. In this way we are able to derive brane descent relations in M-theory from pairs of M9, anti-M9 branes. Then in section 3 we propose the construction of a non-BPS, unstable, M10-brane from which the BPS M9-brane is obtained through tachyonic condensation. Again, this tachyonic mode must be associated to open M2-branes ending on the M10-brane, as required by the duality with type IIA. We thereby present the brane descent relations in terms of this spacetime-filling brane. In section 4 we discuss the existence of other non-BPS unstable branes in M-theory and the possibility of stabilising them within the Horava-Witten construction [14]. In particular, in this picture the tachyonic mode of the non-BPS M10-brane is projected out. Finally section 5 contains some conclusions.

\section{BPS M-branes as bound states}

In this section we show how the M-theory BPS branes can be realised as bound states of $n$ (M9, anti-M9) pairs of branes. As we have mentioned in the introduction we can ignore the couplings of the tachyon in the worldvolume effective action of this system and set the field strength of the antibranes to zero, so that we can simply study the effective action of $n$ coincident M9branes. We use the WZ term constructed recently in [12, but only consider explicitly those couplings that are relevant for the analysis of the topological defects. We also ignore all numerical prefactors and factors of $\alpha^{\prime}$ as well as the explicit couplings to the mass.

The WZ term of the M9-brane effective action contains the couplingsf [12]:

$$
\begin{aligned}
& S_{\mathrm{WZ}}^{(M 9)}=\int_{R^{8+1}}\left[i_{\hat{k}} \hat{B}^{(10)}+i_{\hat{k}} \hat{N}^{(8)} \wedge \hat{\mathcal{F}}+i_{\hat{k}} \hat{\tilde{C}} \wedge \hat{\mathcal{F}} \wedge \hat{\mathcal{F}}+\right. \\
& \left.+\hat{C}_{\hat{\mu}_{1} \hat{\mu}_{2} \hat{\mu}_{3}} D \hat{X}^{\hat{\mu}_{1}} D \hat{X}^{\hat{\mu}_{2}} D \hat{X}^{\hat{\mu}_{3}} \wedge \hat{\mathcal{F}} \wedge \hat{\mathcal{F}} \wedge \hat{\mathcal{F}}+\hat{A} \wedge \hat{\mathcal{F}} \wedge \hat{\mathcal{F}} \wedge \hat{\mathcal{F}} \wedge \hat{\mathcal{F}}+\ldots\right]
\end{aligned}
$$

where the dots indicate the couplings that we have omitted. This action is invariant under the local isometric transformations generated by the Killing

\footnotetext{
${ }^{4}$ Eleven dimensional fields are denoted with hats.
} 
vector $\hat{k}^{\hat{\mu}}$, since the pull-backs onto the worldvolume occur with covariant derivatives (defined below) or contracting with the Killing vector. This vector has to be taken along a worldvolume direction in order to obtain the D8-brane after double dimensional reduction (see [10, [11]). $\hat{B}^{(10)}$ is the spacetime field electric-magnetic dual to the mass, $\hat{N}^{(8)}$ is the dual of the Killing vector considered as a 1-form $\| 15 \rrbracket$, and $\hat{C}, \hat{\tilde{C}}$ are, respectively, the 3 - and 6 -forms of eleven dimensional supergravity. $\hat{\mathcal{F}}$ is the field strength of the worldvolume vector field describing an M2-brane, wrapped on the Killing direction, ending on the brane: $\hat{\mathcal{F}}=d \hat{b}^{(1)}+i_{\hat{k}} \hat{C} \equiv \hat{F}+i_{\hat{k}} \hat{C}$. Finally, $\hat{A} \equiv-\hat{k}^{-2} \hat{k}_{\hat{\mu}} \partial \hat{X}^{\hat{\mu}}$, and the covariant derivatives are defined as: $D \hat{X}^{\hat{\mu}}=\partial \hat{X}^{\hat{\mu}}+\hat{A} \hat{k}^{\hat{\mu}}$ (see [12] and 116, 15] for more details).

The M6-brane, or M-theory Kaluza-Klein monopole, is realised as a bound state (M9, anti-M9) [3]. This can be simply read from the term:

$$
\int_{R^{8+1}} i_{\hat{k}} \hat{N}^{(8)} \wedge \hat{F}
$$

in the M9-brane effective action. As mentioned in the introduction, there must be a tachyonic mode in the spectrum of a wrapped M2-brane stretched between the M9 and the anti-M9, whose condensation will be accompanied by a non-trivial magnetic flux, so that a coupling:

$$
\int_{R^{6+1}} i_{\hat{k}} \hat{N}^{(8)}
$$

remains in the worldvolume. The M-theory Kaluza-Klein monopole is charged with respect to this field [15], so this is the topological defect that remains after the condensation.

The M5-brane is realised, in turn, as an instanton-like configuration. The term:

$$
\int_{R^{8+1}}\left(i_{\hat{k}} \hat{\tilde{C}}\right) \wedge \operatorname{Tr}(\hat{F} \wedge \hat{F})
$$

in the worldvolume effective action of $n$ M9-branes gives a coupling

$$
\int_{R^{4+1}}\left(i_{\hat{k}} \hat{\tilde{C}}\right)
$$

when $\int_{R^{4}} \operatorname{Tr}(\hat{F} \wedge \hat{F})=$ integer, i.e. when the homotopy group $\Pi_{3}(U(n))=Z$. This happens for all $n>2$, however in the particular case $n=2^{k-1}$, where $2 k$

\footnotetext{
${ }^{5}$ Since a single M9-brane contains in its worldvolume a U(1) vector field a system of $n$ coincident M9-branes is described by a $\mathrm{U}(n)$ worldvolume gauge theory. Therefore the corresponding effective action is given by the same expression (2.1) with $F \in \mathrm{U}(n)$ and where the trace is taken over the $\mathrm{U}(n)$ indices.
} 
is defined as the codimension of the topological defect (in this case $k=2$ ), it is possible to give a representation of the tachyon vortex configuration (the generator of $\left.\Pi_{2 k-1}(U(n))\right)$ such that all higher and lower dimensional charges vanish [ [⿴囗十 . For our particular case $n=2$, and the M5-brane is realised as a bound state $\mathrm{M} 5=2$ (M9, anti-M9) [0.

The term responsible for the realisation of the M2-brane as a topological defect is:

$$
\int_{R^{8+1}} \hat{C}_{\hat{\mu}_{1} \hat{\mu}_{2} \hat{\mu}_{3}} D \hat{X}^{\hat{\mu}_{1}} D \hat{X}^{\hat{\mu}_{2}} D \hat{X}^{\hat{\mu}_{3}} \wedge \operatorname{Tr}(\hat{F} \wedge \hat{F} \wedge \hat{F}) .
$$

Now we have $\Pi_{5}(U(4))=Z$, and the topological defect is realised as M2=4 (M9, anti-M9), given that the non-trivial integration of the gauge field gives rise to a coupling:

$$
\int_{R^{2+1}} \hat{C}_{\hat{\mu}_{1} \hat{\mu}_{2} \hat{\mu}_{3}} D \hat{X}^{\hat{\mu}_{1}} D \hat{X}^{\hat{\mu}_{2}} D \hat{X}^{\hat{\mu}_{3}}
$$

which describes an M2-brane with an isometric transverse direction!.

Finally, the term

$$
\int_{R^{8+1}} \hat{A} \wedge \operatorname{Tr}(\hat{F} \wedge \hat{F} \wedge \hat{F} \wedge \hat{F})
$$

describes an M-wave as a bound state of 8 (M9, anti-M9) pairs. In this case $\Pi_{7}(U(8))=Z$, and the field that remains after the condensation: $\hat{A}=$ $-\hat{k}^{-2} \hat{k}_{\hat{\mu}} \partial \hat{X}^{\hat{\mu}}$ is the field to which the M-wave couples minimally [17].

These realisations of M-branes as bound states of M9, anti-M9 pairs are those that one would obtain by oxidising the brane descent relations in the Type IIA theory in terms of D8, anti-D8 pairs of branes. Here we have shown that the M9-brane worldvolume effective action correctly contains the couplings describing these realisations.

In the type IIA theory it is possible to write the brane descent relations in terms of non-BPS spacetime-filling D9-branes, and in this way all the symmetries of the spacetime are preserved [6]. This D9-brane is unstable because the open strings ending on it contain tachyonic excitations. These

\footnotetext{
${ }^{6}$ This definition of the M5-brane seems to imply that a pile of coincident M5-branes would simply be described by a $\mathrm{U}(m)$ gauge theory. This does not contradict the fact that the M5-brane field content must be that of the six dimensional antisymmetric tensor multiplet, whose non-abelian extension is not known, because in the bound state construction the M5-brane is wrapped on the Killing direction of the M9-brane and therefore its field content must be that of the five dimensional vector multiplet.

${ }^{7}$ Since the M2-brane cannot move along this direction, which plays the role of the eleventh coordinate, it behaves effectively as a D2-brane, and a set of such coincident M2-branes can then be described by a $\mathrm{U}(m)$ gauge theory.
} 
modes however can condense in a kink (resp. anti-kink) configuration, giving rise to a D8 (resp. anti-D8) brane as the topological defect. Oxidation to Mtheory predicts then an unstable M10-brane from which the M9-brane should be obtained after tachyonic condensation. In the next section we analyse this possibility.

\section{The non-BPS M10-brane}

Having the action of the M9-brane it is possible to construct the action of a non-BPS M10-brane which gives rise to this brane after tachyonic condensation. In this case, since the field strength $\hat{\mathcal{F}}$ is given by: $\hat{\mathcal{F}}=d \hat{b}^{(1)}+i_{\hat{k}} \hat{C}$, the tachyonic mode must be associated to an M2-brane, wrapped on the Killing direction, ending on the M10-brane.

The clue for the construction of the effective action is to realise that the M10-brane should reproduce the Type IIA non-BPS D9-brane [6] upon double dimensional reduction. The WZ term of the action of type II nonBPS D $p$-branes has been constructed in [18]. In the particular case of the D9-brane it $\operatorname{reads}^{8}$ :

$$
\begin{aligned}
S_{\mathrm{WZ}}^{(D 9)}= & \int_{R^{9+1}}\left[C^{(9)}+C^{(7)} \wedge \mathcal{F}+C^{(5)} \wedge \mathcal{F} \wedge \mathcal{F}+C^{(3)} \wedge \mathcal{F} \wedge \mathcal{F} \wedge \mathcal{F}+\right. \\
& \left.+C^{(1)} \wedge \mathcal{F} \wedge \mathcal{F} \wedge \mathcal{F} \wedge \mathcal{F}\right] \wedge d T
\end{aligned}
$$

where $C^{(p)}$ denotes the $p$-form RR-potential, $\mathcal{F}=F+B^{(2)}$, with $F=d b^{(1)}$ and $B^{(2)}$ the NS-NS 2-form, and $T$ stands for the, real, tachyon field induced in the worldvolume by the open strings ending on the brane. When the tachyon condenses to a non-trivial kink configuration, depending on a single coordinate $x$ and such that $\int d T(x)= \pm 2 T_{0}$, where $\left\{T_{0},-T_{0}\right\}$ are the two minima of the tachyon potential, we have, in the limit of zero size: $d T(x)=$ $2 T_{0} \delta\left(x-x_{0}\right) d x$, and substituting in (3.1) the effective action of a BPS D8brane localised in $x_{0}$ is obtained [18].

The WZ part of the effective action of the proposed non-BPS M10-brane is then given byf:

$$
S_{\mathrm{WZ}}^{(M 10)}=\int_{R^{9+1}}\left[i_{\hat{k}} \hat{B}^{(10)}+i_{\hat{k}} \hat{N}^{(8)} \wedge \hat{\mathcal{F}}+i_{\hat{k}} \hat{\tilde{C}} \wedge \hat{\mathcal{F}} \wedge \hat{\mathcal{F}}+\hat{C}_{\hat{\mu}_{1} \hat{\mu}_{2} \hat{\mu}_{3}}\right.
$$

\footnotetext{
${ }^{8}$ We ignore the contribution of the A-roof genus and, again, all numerical prefactors and factors of $\alpha^{\prime}$.

${ }^{9}$ We use dots to denote those terms that are not relevant for the analysis of the topological defects.
} 


$$
\left.. D \hat{X}^{\hat{\mu}_{1}} D \hat{X}^{\hat{\mu}_{2}} D \hat{X}^{\hat{\mu}_{3}} \wedge \hat{\mathcal{F}} \wedge \hat{\mathcal{F}} \wedge \hat{\mathcal{F}}+\hat{A} \wedge \hat{\mathcal{F}} \wedge \hat{\mathcal{F}} \wedge \hat{\mathcal{F}} \wedge \hat{\mathcal{F}}+\ldots\right] \wedge d \hat{T}
$$

since this action reproduces (3.1) after double dimensional reduction along the Killing direction. Also, the condensation of the tachyon field into a nontrivial kink configuration, $d \hat{T}(x)=2 \hat{T}_{0} \delta\left(x-x_{0}\right) d x$, in the limit of zero size, gives the M9-brane effective action that we considered in the previous section. Like in the non-BPS D9-brane, the tachyon field living in the worldvolume of the M10-brane is a real scalar, in this case induced by open M2-branes wrapped on the Killing direction $\square$.

Regarding the DBI part of the effective action, it has been argued in 20] that non-BPS Dp-branes may be described by a DBI action円:

$$
S_{\mathrm{DBI}}^{(\mathrm{Dp})}=-\int_{R^{p+1}} \sqrt{|\operatorname{det}(G+\mathcal{F})|} R(T, \partial T, \ldots),
$$

where $R$ is some function of the tachyon field vanishing at the minimum of the tachyon potential. In this way the worldvolume action vanishes identically, and can describe a configuration indistinguishable from the vacuum. On the other hand, for vanishing tachyon field $R$ gives a constant, and the action in 20 for a non-BPS Dp-brane involving the massless fields is recovered. For a tachyonic kink configuration in the zero size limit it has been argued that $R(x) \sim C \delta\left(x-x_{0}\right)$ [21], and (3.3) reduces to the DBI part of the effective action of a BPS D(p-1)-brane.

Similarly, we can argue that the DBI part of the non-BPS M10-brane effective action is given by:

$$
S_{\mathrm{DBI}}^{(\mathrm{M} 10)}=-\int_{R^{9+1}}|\hat{k}|^{3} \sqrt{\left|\operatorname{det}\left(\hat{\Pi}+|\hat{k}|^{-1} \hat{\mathcal{F}}\right)\right|} \hat{R}(\hat{T}, \partial \hat{T}, \ldots),
$$

such that when the tachyon condenses to its minimum the DBI effective action of the M9-brane is recovered [10, 11]:

$$
S_{\mathrm{DBI}}^{(\mathrm{M} 9)}=-\int_{R^{8+1}}|\hat{k}|^{3} \sqrt{\left|\operatorname{det}\left(\hat{\Pi}+|\hat{k}|^{-1} \hat{\mathcal{F}}\right)\right|} .
$$

Here $\hat{\Pi}$ is the pull-back of the spacetime metric:

$$
\hat{\Pi}=D \hat{X}^{\hat{\mu}} D \hat{X}^{\hat{\nu}} \hat{g}_{\hat{\mu} \hat{\nu}}=\partial \hat{X}^{\hat{\mu}} \partial \hat{X}^{\hat{\nu}}\left(\hat{g}_{\hat{\mu} \hat{\nu}}+|\hat{k}|^{-2} \hat{k}_{\hat{\mu}} \hat{k}_{\hat{\nu}}\right)
$$

\footnotetext{
${ }^{10}$ The presence of a tachyonic mode in the spectrum of open M2-branes ending on the M10-brane is inferred by duality with the type IIA theory, but being this a strong-weak coupling duality the open M2-branes are intrinsically non-perturbative and the presence of this instability cannot be tested by any perturbative methods.

${ }^{11}$ Here we are only concerned with the bosonic part of the action. See [20] for more details.
} 
The proposed M10-brane action, given by (3.2) and (3.4), contains a Killing direction in its worldvolume. Therefore it is only invariant under ten dimensional Lorentz transformations, consistently with the fact that it is defined in a massive eleven dimensional background. In this sense the M10-brane that we have constructed is spacetime-filling and preserves all the symmetries of the massive eleven dimensional spacetime, including the invariance under local isometric transformations.

Finally, since both the M9 and the anti-M9 branes can be obtained from the M10-brane when the tachyon condenses to a kink or an anti-kink configuration [6], we can write the brane descent relations that we derived in the previous section in terms of spacetime-filling branes as: $\mathrm{M} 9=\mathrm{M} 10, \mathrm{M} 6=2$ $\mathrm{M} 10, \mathrm{M} 5=4 \mathrm{M} 10, \mathrm{M} 2=8 \mathrm{M} 10, \mathrm{M}$-wave=16 M10 [2].

\section{Other non-BPS branes in M-theory}

We have seen how the duality between M-theory and type IIA predicts the existence of an unstable non-BPS M10-brane from which the type IIA D9brane is obtained after double dimensional reduction. Similarly, it is possible to construct unstable non-BPS branes in M-theory giving rise to the whole spectrum of non-BPS D-branes in type IIA. One finds that apart from the M10-brane there are M(-1), M1, M4, M5 and M8 non-BPS branes [3. These branes can be described as bound states of BPS M-branes as: $M p=(M(p+1)$, anti-M(p+1)). In this brane antibrane unstable system the complex tachyonic excitation in the open M2-branes stretched between the brane and the antibrane condenses through a kink configuration, giving rise to the non-BPS M-brane, which is also unstable, because the open M2-branes ending on it contain still a real tachyonic excitation.

The tachyonic excitation in the non-BPS Mp-brane can in turn condense, and give rise to a BPS M-brane. A careful analysis shows that the dimension of this resulting M-brane depends on whether the M2-brane ending on the non-BPS Mp-brane, whose tachyonic mode is condensing, is wrapped or unwrapped 14 . For the M1, M5 and M8 branes the tachyonic mode is associated to a wrapped M2-brane ending on the brane, and one can see that the BPS object that remains after the condensation must be an M0, a wrapped M5

\footnotetext{
${ }^{12}$ The WZ part of the effective action describing the set of $n$ coincident M10-branes is of the same form (3.2) but now $\hat{F} \in \mathrm{U}(n)$, the tachyon transforms in the adjoint representation [6] and traces over the $\mathrm{U}(n)$ indices are taken.

${ }^{13}$ Among these branes, the M(-1), M1, M4 and M5 can exist as well in massless M-theory.

${ }^{14}$ As explained in [3 certain realisations of branes as solitons in brane anti-brane systems require to choose one special direction.
} 
and an M6 brane respectively. In the case of the non-BPS M4-brane the tachyonic mode is associated to unwrapped M2-branes ending on the brane, and the object that remains after the condensation is an M2-brane.

Thus, the existence of these non-BPS branes in M-theory allows the construction of BPS branes from brane antibrane pairs in two steps. First, the complex tachyonic mode of the brane stretched between the brane and the antibrane condenses, giving rise to a non-BPS M-brane, with a real tachyonic mode associated to open M2-branes ending on it. Second, this tachyonic mode condenses in the same type of configuration and a stable BPS M-brane emerges. This generalises to M-theory the two-step construction of type II theories of [19, 22].

It is interesting to note that the non-BPS M-branes that we have considered give rise, upon reduction, not only to non-BPS Dp-branes but also to some additional non-BPS branes in the type IIA theory. These branes predict in turn the existence of similar non-BPS branes in type IIB by T-duality. We have mentioned in the introduction that BPS NS-NS and gravitational branes [23] in the type IIA and type IIB theories can be interpreted as solitons in brane antibrane systems [2, 3], with tachyonic condensing charged objects that are extended and non-perturbative. In the realisations discussed in these references the complex tachyonic mode condenses in a vortex-like configuration, and the resulting solitonic object is stable and carries NS-NS or gravitational charge. One could instead consider a two-step construction of these BPS branes as in [19, 22], and this would lead to new intermediate non-BPS "NS-NS" and "gravitational" branes" as remnants of tachyonic kink configurations. These are the additional non-BPS branes that are derived from M-theory.

\subsection{Stable non-BPS M-branes in the Hor̆ava-Witten construction}

Unstable non-BPS branes can become stable when the theory is orbifolded/ orientifolded by an appropriate symmetry (see [1]). Therefore, it is interesting to analyse whether the unstable non-BPS M-branes that we have discussed can become stable in the framework of the Horava-Witten construction [14. One can predict stable non-BPS branes in this construction by uplifting the stable non-BPS branes of the type I' theory, which have in turn been analysed in [24 by using the T-duality connection with type I.

The spectrum of stable non-BPS type I' branes consists on a D(-1), D0,

\footnotetext{
${ }^{15} \mathrm{NS}-\mathrm{NS}$ and gravitational in the sense that they are derived in the two-step construction of this kind of branes, but it is clear that they do not carry any charge.
} 
D1, D6, D7, D8 and D9 branes, where the D0, D1, D8 and D9 are stretched in the interval These branes can be constructed as bound states: $\mathrm{D}(-1)=(\mathrm{D} 0$, anti-D0), D0 $=(\mathrm{D} 0$, anti-D0) (in this case the D0's are 'stretched'), D1 $=(\mathrm{D} 2$, anti-D2) (stretched), D6 $=(\mathrm{D} 6$, anti-D6), D7 $=(\mathrm{D} 8$, anti-D8), D8 $=(\mathrm{D} 8$, antiD8) (stretched). Uplifting these configurations to M-theory we find:

$\mathrm{M}(-1)=(\mathrm{M} 0$, anti-M0), $\mathrm{M} 0=(\mathrm{M} 0$, anti-M0) ('stretched'), $\mathrm{M} 1=(\mathrm{M} 2$, anti-M2) (stretched), M6 $=(\mathrm{M} 6$, anti-M6), M8 $=(\mathrm{M} 9$, anti-M9), M9=(M9, anti-M9) (stretched), together with a stretched M10-brane.

We can thus conclude that the non-BPS M(-1), M1, M8 and M10 branes of uncompactified M-theory are stabilised when one considers M-theory in the interval. Moreover, we find additional branes realised as BPS brane antibrane pairs in which the tachyonic mode is removed from the spectrum by the orbifold projection, and no condensation occurs. This happens for the M0, M6 and M9 non-BPS branes.

The Hor̆ava-Witten construction can be described as M-theory orientifolded by $I_{10} \hat{\Omega}$ (see [26]), where $I_{10}: x^{10} \rightarrow-x^{10}$ and $\hat{\Omega}$ is the operation that reverses the orientation of the M2 and the M5 branes. In this description the end of the world branes of Hor̆ava-Witten are identified as the two fixed planes associated to the orientifold projection with 16 M9-branes on top of them. The connection with the type I' theory through compactification on $S^{1}$ implies that the M6, M8, M9 and M10 branes must become unstable due to the presence of a tachyonic mode in the open M2-branes with one end on the brane and the other in one of the $16+16$ M9-branes located on the top of the orientifold fixed planes.

\section{Conclusions}

We have shown how the brane descent relations that one expects to find in Mtheory by oxidation from Type IIA are indeed predicted by the analysis of the topological defects that can occur in the worldvolume of $n$ M9, anti-M9 pairs of branes. We have proposed as well a non-BPS, unstable, M10-brane, from which the brane descent relations can be expressed in terms of spacetimefilling branes, preserving all the symmetries of the spacetime. Since massive eleven dimensional supergravity is at present only known explicitly when the spacetime contains a Killing isometry [9], the corresponding M10-brane contains as well this Killing isometry in its worldvolume, and therefore it is

\footnotetext{
${ }^{16}$ The D6, D7, D8 and D9 branes are however unstable due to the presence of a tachyonic mode in the open strings with one end on the brane and the other in one of the $16+16$ D8-branes located on top of the orientifold fixed planes. This can be inferred from the type I case 25.
} 
not fully eleven dimensional Lorentz invariant, consistently with the type of eleven dimensional spacetime in which it is defined.

Using the relation between ordinary and higher K-theory groups (see [6], [27): $\tilde{K}\left(S^{10-p}\right)=K^{-1}\left(S^{9-p}\right)$, it is inferred that the branes that can be obtained in the descent construction in this massive M-theory are classified according to:

$$
\tilde{K}\left(S^{10-p}\right)=\left\{\begin{array}{cccc}
Z & \text { for } & p & \text { even } \\
0 & \text { for } & p & \text { odd }
\end{array} .\right.
$$

Therefore the K-theory groups predict the M-wave, M2 and M6 branes ${ }^{[7}$, and an M4 and M8 branes, which are identified as wrapped M5 and M9 branes, identification that is supported by the bound state analysis that we have carried out in this paper.

We have discussed as well the existence of other non-BPS branes in M-theory, giving rise to type IIA non-BPS D-branes after dimensional reduction. These M-branes predict unstable non-BPS "NS-NS" and "gravitational" branes in both the type IIA and type IIB theories, which would arise naturally in a two-step construction, as in [19, 22], of NS-NS and gravitational BPS branes.

Finally, we have shown that when M-theory is compactified in the interval some of the non-BPS M-branes become stable, in particular the spacetimefilling M10-brane, since they give rise to stable non-BPS branes in type I' after reduction. The K-theory classification of stable M-branes in the Horava-Witten picture seems to be given by the K-theory group: $K R\left(S^{9-p} \times\right.$ $S^{1,1}, S^{1,1}$, which provides the M-theory interpretation of the type I' K-theory group [4, 6, 24]: $K R^{-1}\left(S^{8-p} \times S^{1,1}, S^{1,1}\right)$. Some of the non-BPS branes predicted by this group become however unstable due to the presence of background M9-branes in the Horava-Witten picture, which, as in the type I case [25], do not play any role in the K-theory classification.

\section{Acknowledgements}

L. H. would like to acknowledge the support of the European Commission TMR programme grant ERBFMBICT-98-2872, and the Theory Division at CERN, where this work has been completed, for hospitality. The I.C. Theory Group is supported by PPARC under SPG grant 613.

\footnotetext{
${ }^{17}$ For the M-wave and the M6 the $S^{1}$ direction coincides with their special isometric direction.
} 


\section{References}

[1] A. Sen, Non-BPS States and Branes in String Theory, hep-th/9904207;

A. Lerda and R. Russo, Stable Non-BPS States in String Theory: a Pedagogical Review, hep-th/9905006; J. H. Schwarz, TASI Lectures on Non-BPS D-Brane Systems, hep-th/9908144; O. Bergman and M. Gaberdiel, NonBPS Dirichlet Branes, hep-th/9908126.

[2] P. Yi, Membranes from Five-Branes and Fundamental Strings from Dpbranes, Nucl. Phys. 550 (1999) 214, hep-th/9901159.

[3] L. Houart and Y. Lozano, Type II Branes from Brane-Antibrane in Mtheory, Nucl. Phys. B (to appear), hep-th/9910266.

[4] E. Witten, D-Branes and K-Theory, J. High Energy Phys. 9812 (1998) 019, hep-th/9810188.

[5] L. Houart and Y. Lozano, S-Duality and Brane Descent Relations, hep-th/9911173.

[6] P. Hor̆ava, Type IIA D-Branes, K-Theory and Matrix Theory, Adv. Theor. Math. Phys. 2 (1999) 1373, hep-th/9812135.

[7] K. Bautier, S. Deser, M. Henneaux and D. Seminara, No Cosmological D=11 Supergravity, Phys. Lett. B406 (1997) 49, hep-th/9704131.

[8] L.J. Romans, Massive N=2a Supergravity in Ten Dimensions, Phys. Lett. B169 (1986) 374.

[9] E. Bergshoeff, Y. Lozano and T. Ortín, Massive Branes, Nucl. Phys. B518 (1998) 363, hep-th/9712115.

[10] E. Bergshoeff and J.P. van der Schaar, On M9-branes, Class. Quant. Grav. 16 (1999) 23, hep-th/9806069.

[11] E. Eyras and Y. Lozano, Brane Actions and String Dualities, hep-th/9812225.

[12] T. Sato, A 10-form Gauge Potential and an M-9-brane Wess-Zumino Action in Massive 11D Theory, hep-th/9912030.

[13] C. Kennedy and A. Wilkins, Ramond-Ramond Couplings on BraneAntibrane Systems, Phys. Lett. B464 (1999) 206, hep-th/9905195.

[14] P. Hor̆ava and E. Witten, Heterotic and Type I String Dynamics from Eleven Dimensions, Nucl. Phys. B460 (1996) 506, hep-th/9510209. 
[15] E. Bergshoeff, E. Eyras and Y. Lozano, The Massive Kaluza-Klein Monopole, Phys. Lett. B430 (1998) 77, hep-th/9802199.

[16] E. Bergshoeff, B. Janssen and T. Ortín, Kaluza-Klein Monopoles and Gauged Sigma-Models, Phys. Lett. B410 (1997) 132, hep-th/9706117.

[17] E. Bergshoeff and P.K. Townsend, Super D-branes, Nucl. Phys. B490 (1997) 145, hep-th/9611173.

[18] M. Billó, B. Craps and F. Roose, Ramond-Ramond couplings of non-BPS D-branes, J. High Energy Phys. 9906 (1999) 033, hep-th/9905157.

[19] A. Sen, BPS D-branes on Non-supersymmetric Cycles, J. High Energy Phys. 9812 (1998) 021, hep-th/9812031.

[20] A. Sen, Supersymmetric World-volume Action for Non-BPS D-branes, J. High Energy Phys. 9910 (1999) 008, hep-th/9909062.

[21] J. Klusoŭ, Remark about Non-BPS Brane in IIA Theory, hep-th/9909194.

[22] A. Sen, SO(32) Spinors of Type I and other Solitons on Brane-Antibrane Pairs, J. High Energy Phys. 9809 (1998) 023, hep-th/9808141.

[23] C.M. Hull, Gravitational Duality, Branes and Charges, Nucl. Phys. B509 (1998) 216, hep-th/9705162.

[24] O. Bergman, E.G. Gimon and P. Hor̆ava, Brane Transfer Operations and T-Duality of Non-BPS States, J. High Energy Phys. 9904 (1999) 010, hep-th/9902160.

[25] M. Frau, L. Gallot, A. Lerda and P. Strigazzi, Stable non-BPS D-branes in Type I String Theory, Nucl. Phys. B (to appear), hep-th/9903123.

[26] E. Bergshoeff, E. Eyras, R. Halbersma, C.M. Hull, Y. Lozano and J.P. van der Schaar, Spacetime-filling Branes and Strings with Sixteen Supercharges, Nucl. Phys. B564 (2000) 29, hep-th/9812224.

[27] K. Olsen and R. Szabo, Constructing D-Branes from K-Theory, hep-th/9907140. 